\title{
Assessment of Undiscovered Oil and Gas Resources of the Powder River Basin Province of Wyoming and Montana- 2006 Update
}

Using a geology-based assessment method, the U.S. Geological Survey estimated means of 16.6 trillion cubic feet of undiscovered natural gas, 639 million barrels of undiscovered oil, and 131 million barrels of natural gas liquids in the Powder River Basin Province.

\section{Introduction}

The U.S. Geological Survey (USGS) has completed an update of the 1995 assessment (USGS Fact Sheet FS-146-02) of the potential for undiscovered resources in conventional oil and gas accumulations of the Powder River Basin Province of northeastern Wyoming and southeastern Montana (fig. 1). The 2002 assessment of continuous (unconventional) oil and gas accumulations were not reassessed. The current assessment of conventional oil and gas resources, based on geologic elements such as hydrocarbon source rocks (source rock maturation and hydrocarbon generation and migration), reservoir rocks (sequence stratigraphy and petrophysical properties), and hydrocarbon traps (trap formation and timing), includes five Total Petroleum Systems (TPS) identified in the province by the USGS; eight assessment units (AU) were defined within the TPSs. Estimates of the undiscovered conventional oil and gas resource in these eight AUs, in addition to the estimates of undiscovered continuous oil and gas resources within the province that were reported in USGS Fact Sheet FS-146-02 are presented in table 1 .

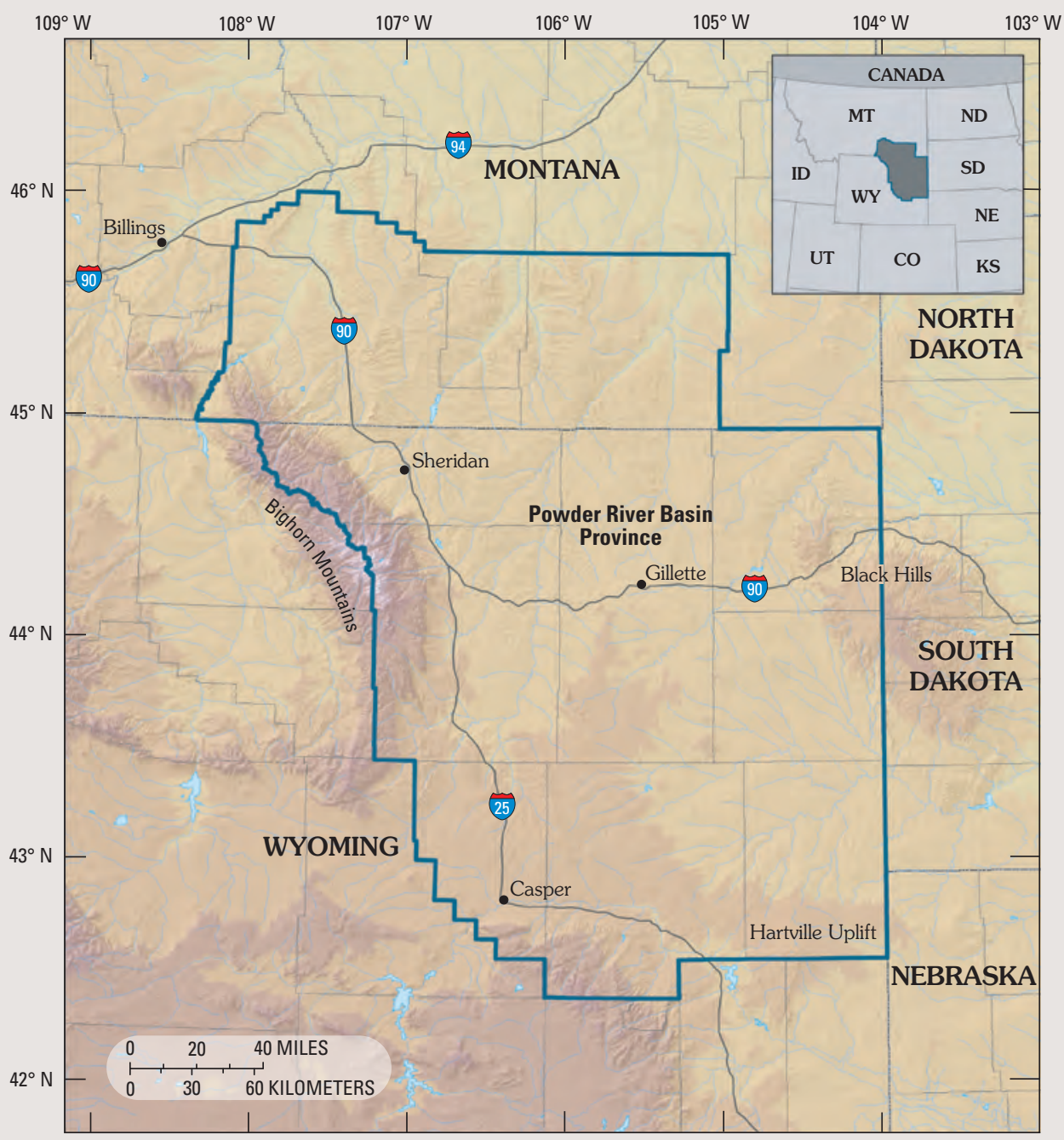

Figure 1. Powder River Basin Province of northeastern Wyoming and southeastern Montana.

\section{Resource Summary}

The USGS estimated means of 16.6 trillion cubic feet of gas, 639 million barrels of oil, and 131 million barrels of total natural gas liquids for undiscovered continuous and conventional resources in the Powder River Basin Province (table 1). Most of the undiscovered gas resource (93 percent, or 15.5 trillion cubic feet) is continuous. Of the 1.16 trillion cubic feet of conventional gas at the mean, about 91 percent, or 1.05 trillion cubic feet is estimated to be from the Fall River-Lakota and Muddy Sandstones AUs of the Mowry TPS. Of the 215 million barrels of conventional oil at the mean: (1) about 30 percent, or 64 million barrels, is estimated to be from the Fall River-Lakota Sandstone AU; (2) about 28 percent, or 61 million barrels, is estimated to be from the MinnelusaTensleep-Leo Sandstones AU of the Pennsylvanian-Permian TPS; and (3) about 
Table 1. Powder River Basin Province Assessment Results.

[MMBO, million barrels of oil; BCFG, billion cubic feet of gas; MMBNGL, million barrels of natural gas liquids.Results shown are fully risked estimates. For gas accumulations, all liquids are included as NGL (natural gas liquids). F95 denotes a 95 percent chance of at least the amount tabulated; other fractiles are defined similarly. Fractiles are additive under the assumption of perfect positive correlation. TPS, Total Petroleum System; AU, Assessment Unit. Gray shading indicates not applicable.]

\begin{tabular}{|c|c|c|c|c|c|c|c|c|c|c|c|c|c|}
\hline \multirow{2}{*}{$\begin{array}{l}\text { Total Petroleum Systems } \\
\text { (TPS) } \\
\text { and Assessment Units (AU) }\end{array}$} & \multirow{2}{*}{$\begin{array}{l}\text { Field } \\
\text { type }\end{array}$} & \multicolumn{12}{|c|}{$\begin{array}{c}\text { Total undiscovered resources } \\
\text { Gas (BCFG) }\end{array}$} \\
\hline & & F95 & F50 & F5 & Mean & F95 & F50 & F5 & Mean & F95 & F50 & F5 & Mean \\
\hline \multicolumn{14}{|l|}{$\begin{array}{l}\text { Pennsylvanian-Permian } \\
\text { Composite TPS }\end{array}$} \\
\hline \multirow{2}{*}{ Minnelusa-Tensleep-Leo AU } & Oil & 21.38 & 56.48 & 112.13 & 60.51 & 0.87 & 2.52 & 5.85 & 2.83 & 0.02 & 0.08 & 0.25 & 0.10 \\
\hline & Gas & & & & & 3.34 & 7.26 & 13.39 & 7.32 & 0.17 & 0.41 & 0.86 & 0.44 \\
\hline \multicolumn{14}{|l|}{ Mowry TPS } \\
\hline \multirow{2}{*}{ Fall River-Lakota Sandstone AU } & Oil & 18.14 & 58.58 & 127.53 & 64.05 & 18.58 & 64.98 & 162.77 & 74.70 & 1.03 & 3.81 & 10.25 & 4.48 \\
\hline & Gas & & & & & 104.85 & 507.46 & $1,257.06$ & 574.51 & 9.72 & 49.36 & 131.97 & 57.47 \\
\hline \multirow{2}{*}{ Muddy Sandstone AU } & Oil & 10.95 & 40.62 & 106.81 & 47.34 & 31.03 & 122.43 & 360.08 & 149.14 & 2.71 & 10.89 & 32.87 & 13.43 \\
\hline & Gas & & & & & 52.61 & 214.07 & 564.62 & 248.77 & 4.93 & 20.79 & 58.58 & 24.85 \\
\hline \multicolumn{14}{|l|}{ Niobrara TPS } \\
\hline Frontier-Turner Sandstones AU & Oil & 2.46 & 9.12 & 21.37 & 10.18 & 8.72 & 34.44 & 93.45 & 40.47 & 0.59 & 2.41 & 6.97 & 2.91 \\
\hline Sussex-Shannon Sandstones AU & Oil & 2.75 & 7.97 & 16.98 & 8.67 & 2.25 & 7.09 & 17.30 & 8.09 & 0.17 & 0.55 & 1.45 & 0.65 \\
\hline Mesaverde-Lewis Sandstones AU & Oil & 1.55 & 5.19 & 13.20 & 6.00 & 1.90 & 6.97 & 19.84 & 8.41 & 0.13 & 0.48 & 1.44 & 0.59 \\
\hline \multicolumn{14}{|l|}{$\begin{array}{l}\text { Tertiary-Upper Cretaceous } \\
\text { Coalbed Methane TPS }\end{array}$} \\
\hline $\begin{array}{l}\text { Eastern Basin Margin Upper } \\
\text { Ft. Union Sandstone AU } \\
\end{array}$ & Gas & & & & & 0.00 & 0.00 & 107.43 & 27.37 & 0.00 & 0.00 & 0.00 & 0.00 \\
\hline \multicolumn{14}{|l|}{ Paleozoic-Mesozoic TPS } \\
\hline Basin Margin AU & Oil & 4.79 & 16.30 & 36.76 & 17.93 & 3.46 & 12.58 & 32.89 & 14.66 & 0.11 & 0.44 & 1.23 & 0.53 \\
\hline $\begin{array}{l}\text { Total Conventional } \\
\text { Resources }\end{array}$ & & 62.02 & 194.26 & 434.78 & 214.68 & 227.61 & 979.80 & $2,634.68$ & $1,156.27$ & 19.58 & 89.22 & 245.87 & 105.45 \\
\hline \multicolumn{14}{|l|}{$\begin{array}{l}\text { Tertiary-Upper Cretaceous } \\
\text { Coalbed Methane TPS }\end{array}$} \\
\hline Wasatch Formation AU & CBG & & & & & $1,011.94$ & $1,815.71$ & $3,257.89$ & $1,934.09$ & 0.00 & 0.00 & 0.00 & 0.00 \\
\hline Upper Fort Union Formation AU & CBG & & & & & $7,232.13$ & $11,635.87$ & $18,721.10$ & $12,132.50$ & 0.00 & 0.00 & 0.00 & 0.00 \\
\hline $\begin{array}{l}\text { Lower Fort Union-Lance } \\
\text { Formation AU } \\
\end{array}$ & $C B G$ & & & & & 0.00 & 171.67 & 440.90 & 197.90 & 0.00 & 0.00 & 0.00 & 0.00 \\
\hline \multicolumn{14}{|l|}{ Mowry TPS } \\
\hline Mowry Continuous 0il AU & Oil & 116.99 & 189.32 & 306.38 & 197.61 & 103.35 & 185.50 & 332.95 & 197.61 & 5.56 & 10.91 & 21.37 & 11.86 \\
\hline \multicolumn{14}{|l|}{ Niobrara TPS } \\
\hline Niobrara Continuous Oil AU & Oil & 135.53 & 217.49 & 349.03 & 226.67 & 119.54 & 213.10 & 379.87 & 226.67 & 6.43 & 12.53 & 24.40 & 13.60 \\
\hline \multicolumn{14}{|l|}{ Cretaceous Biogenic Gas TPS } \\
\hline $\begin{array}{l}\text { Shallow Continuous } \\
\text { Biogenic Gas AU }\end{array}$ & Gas & & & & & 341.92 & 712.15 & $1,483.26$ & 786.64 & 0.00 & 0.00 & 0.00 & 0.00 \\
\hline $\begin{array}{l}\text { Total Continuous } \\
\text { Resources }\end{array}$ & & 252.52 & 406.81 & 655.41 & 424.28 & $8,808.88$ & $14,734.00$ & $24,615.97$ & $15,475.41$ & 11.99 & 23.44 & 45.77 & 25.46 \\
\hline $\begin{array}{l}\text { Total Undiscovered } \\
\text { Oil and Gas Resources }\end{array}$ & & 314.54 & 601.07 & $1,090.19$ & 638.96 & $9,036.49$ & $15,713.80$ & $27,250.65$ & $16,631.68$ & 31.57 & 112.66 & 291.64 & 130.91 \\
\hline
\end{tabular}

22 percent, or 47 million barrels is estimated to be from the Muddy Sandstone AU (table 1).

The Frontier-Turner, Sussex-Shannon, and Mesaverde-Lewis Sandstones AUs from the Niobrara TPS are estimated to contain means of 25 million barrels of conventional oil (12 percent) and 57.0 billion cubic feet of gas ( 5 percent) of the total undiscovered conventional resource (table 1).
The Basin Margin AU of the

Paleozoic-Mesozoic TPS is estimated to contain a mean of 18 million barrels and 14.7 billion cubic feet of total undiscovered conventional resource (table 1).

The Eastern Basin Margin-Upper Ft. Union Sandstone AU of the TertiaryUpper Cretaceous Coalbed Methane TPS is estimated to contain a mean of 27.4 billion cubic feet of total undiscovered conventional gas resource (table 1).

\section{For Further Information}

Supporting geologic studies and reports on the assessment method used in the Powder River Basin Province assessments of conventional resources are in preparation (2006). Assessment results are available at the USGS Central Energy Team website, http://energy.cr.usgs.gov/ oilgas/noga.

\section{Powder River Basin Province Assessment Team}

Lawrence O. Anna (Task Leader; lanna@ usgs.gov), Ronald R. Charpentier, Troy A. Cook, Timothy R. Klett, Richard M. Pollastro, and Christopher J. Schenk. 